\title{
FORMULACIÓN DE UN MODELO METODOLÓGICO PARA LA IMPLEMENTACIÓN DEL PLAN INSTITUCIONAL DE GESTIÓN AMBIENTAL - PIGA - EN LOS CEAD Y CERES DE LA UNIVERSIDAD NACIONAL ABIERTA Y A DISTANCIA, UNAD
}

\author{
William Ignacio Montealegre Torres ${ }^{102}$ \\ RESUMEN
}

A través del séptimo objetivo de desarrollo, la Declaración del Milenio incorporó la sostenibilidad ambiental dentro de las prioridades. Su inclusión se dio así en reconocimiento no sólo del valor intrínseco del medio ambiente, sino también de su importancia para la superación de la pobreza, la salud, la igualdad de género y los demás componentes del bienestar humano.

La Universidad Nacional Abierta y a Distancia, UNAD, en el artículo primero de la política ambiental ha establecido que se propenderá por el mejoramiento continuo de las condiciones ambientales en sus instalaciones, elevando los niveles de salud y bienestar de la comunidad mediante el cumplimiento responsable de las disposiciones legales, el aporte a la formación a distancia y en ambientes virtuales, la investigación, la proyección social y la gestión en aspectos relacionados con el buen manejo de las sedes de la Institución, el uso eficiente de los recursos, el reciclaje y la reutilización de los materiales, y el tratamiento y la disposición adecuada de los residuos.

La Escuela de Ciencias Agrícolas, Pecuarias y del Medio Ambiente (ECAPMA), del CEAD Pitalito, en cumplimiento de esos mandatos institucionales y legales, con el apoyo de SIUNAD, viene formulando un modelo metodológico para la implementación del Plan Institucional de Gestión Allmbiental (PIGA) en este CEAD y que sea un aporte para los CEAD, CERES y CCAV de la UNAD.

El proyecto tiene tres objetivos específicos: caracterizar el entorno físico —químico, biótico y social- económico para valorar las condiciones ambientales externas de la institución; Identificar y evaluar el impacto ambiental para valorar las condiciones ambientales internas de la institución y analizar el uso y manejo de los recursos agua, residuos sólidos, energía eléctrica, insumos, al interior de la Institución, para su valoración y consideración dentro de los programas del PIGA.

Se utilizaron cuatro técnicas de investigación: la observación, la encuesta, análisis de información secundaria e información primaria y el método fue el inductivo. Mediante el análisis de la información, para cada uno de los recursos, se constituyen los programas del PIGA y se establecen unos indicadores

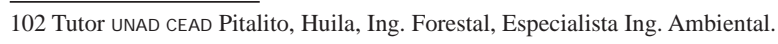


Palabras clave: Plan de Gestión Ambiental, método inductivo, calificación ambiental, matriz de impacto ambiental.

\begin{abstract}
Across the seventh aim of development, the Declaration of the Millenium incorporated the environmental sustainability inside the priorities. Its incorporation was given in recognition not only of the intrinsic value of the environment, but also of his importance for the overcoming of the poverty, the health, the equality of kind and other components of the human well-being.
\end{abstract}

The National Open University and Distance UNAD, in the first article of the environmental policy as established that will be tended by the constant improvement of the environmental conditions in his facilities, raising standards of health and well-being of the community by implementing responsible of the laws, the contribution to the formation distantly and in virtual environments, research, the social projection and the management in aspects related to the good managing of the headquarters of the Institution, the efficient use of the resources, the recycling and the reutilization of the materials, and the treatment and the suitable disposition of the residues.

The School of Agricultural Sciences, Livestock and the Environment (ECAPMA) of Pitalito CEAD, in compliance with these legal and institutional mandates, with the support of Siuna, is crafting a methodological model for the implementation of Environmental Management Institutional Plan (PIGA ) in the CEAD and is a contribution to the CEAD, CERES and the UNAD CCAV.

Three specific objectives: To characterize the physical environment — chemical, biotic and social- economical to assess the external environmental conditions of the institution; Identification and environmental impact assessment to assess the environmental conditions inside the institution and Analyze the use and management of water resources, solid waste, electricity, supplies, within the Bank for review and consideration within the PIGA programs.

With the use of four techniques of research: observation, survey, analysis of secondary information and primary information, applies the inductive method, which allows us to draw general conclusions about something particular. Through analysis of information, for each of the resources, constitute PIGA programs and indicators are identified

Key words: Plan Environmental Management, inductive method, environmental qualification, environmental impact matrix. 


\section{INTRODUCCION}

Comprometidos con los principios del desarrollo sostenible, la UNAD, la Escuela de Ciencias Agrícolas, Pecuarias y del Medio Ambiente del CEAD Pitalito y el Grupo de investigación INYUMACIZO, han impulsado la cátedra y la investigación con los estudiantes hacia el manejo sostenible de los recursos naturales renovables. Este enfoque, ha permitido impulsar procesos de gran significancia para el desarrollo de la región. En este espacio, me permito presentar un proyecto, de gran interés para la UNAD, que ha despertado expectativa en el Departamento del Huila y en el municipio de Pitalito y reivindican el liderazgo del CEAD Pitalito cuando de cuestiones ambientales se trata, denominado: Formulación de un modelo metodológico para la implementación del plan institucional de gestión ambiental - PIGA - en los CEAD y CERES de la Universidad Nacional Abierta y a Distancia UNAD.

El origen del trabajo de investigación se puede circunscribir a los esfuerzos realizados por la Alcaldía mayor de Bogotá desde 2005, al consolidar un primer PIGA y proponer a las instituciones oficiales su formulación, para dar cumplimiento a la normatividad ambiental.

El presente trabajo de investigación pretende formular una metodología que permita, de manera ágil y oportuna al CEAD Pitalito, conocer su realidad ambiental interna y la correspondencia externa basados en el compromiso institucional y propender, mediante programas y proyectos con sus correspondientes indicadores, a mejorar las condiciones ambientales y a liderar, en la zona sur del Huila, estos compromisos. Esta metodología en construcción, debe ser replicable en los CEAD, CERES Y CCAV de la UNAD.

\section{OBJETIVO GENERAL}

Formular un modelo metodológico para la implementación del Plan Institucional de Gestión Ambiental (PIGA), de la Universidad Nacional Abierta y a Distancia UNAD CEAD Pitalito.

\section{OBJETIVOS ESPECÍFICOS}

Caracterizar el entorno físico - químico, biótico y social - económico para valorar las condiciones ambientales externas de la institución.

Identificación y evaluación de impacto ambiental para valorar las condiciones ambientales internas de la institución.

Analizar el uso y manejo de los recursos agua, residuos sólidos, energía eléctrica, insumos, al interior de la Institución para su valoración y consideración dentro de los programas del PIGA. 
ReVISTA DE INVEStigaciones UNAD

\section{METODOLOGÍA}

Es importante reconocer que el sujeto de la gestión ambiental es el ser humano y sus condiciones de salud y bienestar, y que por ende, las acciones que se propongan deben reflejarse en la reducción de los posibles factores de riesgo que se detectan en el diagnóstico. En la presente investigación se han tenido en cuenta diferentes aspectos para el desarrollo metodológico, los cuales sirven de base y soporte, así como guía para el logro de los objetivos propuestos.

Para el logro de los objetivos específicos del proyecto, se desarrolla la metodología que se presenta en la figura 1.

Figura 1. Metodología desarrollada en la formulación del PIGA UNAD CEAD Pitalito, 2010

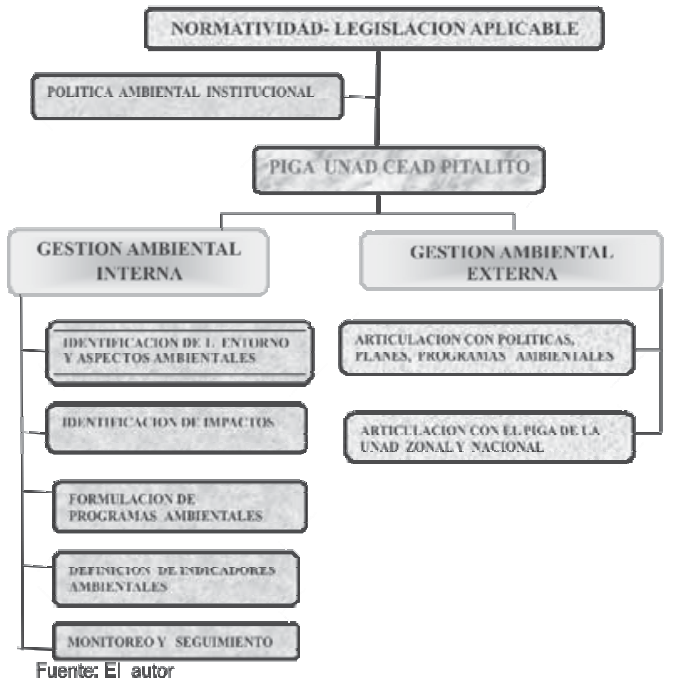

TIPO DE ESTUDIO

La formulación de modelo metodológico del PIGA, es de tipo descriptivo porque "delimita los hechos que conforman el problema de investigación, establece características demográficas de unidades investigadas, identifica formas y conductas de la comunidad académica y administrativa del CEAD Pitalito, y establece comportamientos concretos y específicos de los aspectos que involucran la investigación" (Méndez, 2001). 


\section{MÉTODOS DE INVESTIGACIÓN}

En el presente trabajo se hizo aplicación de los métodos deductivo e inductivo.

Método inductivo. Permite partir de la observación de fenómenos o situaciones particulares que enmarcan el problema de investigación y concluir proposiciones y a su vez, premisas que expliquen fenómenos similares al analizado. Así, los resultados obtenidos pueden ser la base teórica sobre la cual se fundamenten observaciones, descripciones y explicaciones posteriores de realidades con rasgos y características semejantes a la investigada, concretamente, para los demás consumidores.

Método Deductivo. Este método parte de lo general a lo específico y se emplea después del método inductivo, al cual está íntimamente unido, constituyen así dos fases de un mismo proceso.

\section{Enfoque investigativo}

Observación Participativa: permite ir a los protagonistas, participar en su construcción y a su vez permite coconstruir a partir de los encuentros en el contexto por medio de la reflexión de las relaciones que se presentan entre la práctica integrada y la construcción de significados, participando del proceso de construcción, descubriendo el sentido, la dinámica y los procesos de los acontecimientos que viven los protagonistas en el medio en que se desarrolla como practicante de la prueba piloto, permitiendo la descripción de la cultura estudiantil desde el punto de vista de los protagonistas.

Para describir la situación analizada se dedica a descubrir el sentido, la dinámica de los procesos, de los actos, de los acontecimientos y de las afirmaciones textuales de los protagonistas, estas relaciones descriptivas aportan información sobre las situaciones en que se mueven y las percepciones que tienen los protagonistas sobre la situación en que viven, también tiene en cuenta las expectativas y las necesidades de estos.

\section{Fuentes y técnicas para recolección de información}

Para el desarrollo de la presente investigación se hizo acopio de información proveniente tanto de fuentes primarias como secundarias y se aplicó la técnica de la encuesta.

Universo o población: constituye la totalidad de un grupo de elementos u objetos que se quiere investigar, es el conjunto de todos los casos que concuerdan con lo que se pretende investigar. El universo, para este ejercicio piloto es la UNAD CEAD Pitalito.

\section{Tratamiento de la información}

La información recolectada fue sistematizada y organizada en tablas para poder obtener los

datos totales de respuesta a cada pregunta planteada en las encuestas; con el resultado de los datos se elaboraron gráficos y figuras a fin de poder presentar la información de una forma 
sencilla y didáctica con explicaciones en lenguaje sencillo para un fácil entendimiento del público en general.

Luego de obtener los datos generales de las encuestas, se realizó un análisis profundo de acuerdo con el objetivo planteado para la investigación, teniendo presente que este trabajo debe servir de apoyo en la toma de decisiones al CEAD Pitalito, para la puesta en marcha del PIGA.

\section{Población y muestra}

Para determinar el tamaño de la muestra se tuvo en cuenta la población total de la comunidad del CEAD Pitalito (868 personas), para una probabilidad del 50\%, confianza del 95\%, y un error admisible del $5 \%$.

\section{Selección del diseño apropiado para la investigación}

Para el desarrollo de la investigación, se ha seleccionado el diseño no experimental de investigación, que es aquel que se efectúa sin la manipulación intencionada de variables, lo que aquí se realiza es la observación de fenómenos en su ambiente natural.

Universo o población: constituye la totalidad de un grupo de elementos u objetos que se quiere investigar, es el conjunto de todos los casos que concuerdan con lo que se pretende investigar. El universo, para este ejercicio piloto, es la UNAD, CEAD Pitalito.

\section{Procedimientos para la recopilación de datos}

- La observación: es el procedimiento empírico por excelencia, el más antiguo; consiste básicamente en utilizar los sentidos para observar los hechos, realidades sociales y a las personas en su contexto cotidiano. Para que dicha observación tenga validez es necesario que sea intencionada e ilustrada (con un objetivo determinado y guiada por un cuerpo de conocimiento). Se tomaron los datos del número de vehículos que ingresaron a la universidad en diferentes días y horas de acuerdo con el formato diseñado para este fin, el estado de las zonas verdes, de los sistemas eléctrico, alcantarillado, acueducto y residuos sólidos.

De la misma manera, el estado de la infraestructura física, área por persona, ventilación, humedad, olores, ruido, contaminación del aire, iluminación, ubicación de publicidad, tanto interna como externa al CEAD Pitalito.

- La entrevista: la entrevista consiste en una conversación entre dos o más personas, sobre un tema determinado, de acuerdo con ciertos esquemas o pautas determinadas. Entrevistas directas a compañeros tutores, estudiantes, administrativos y visitantes casuales.

- La encuesta: se realizó una encuesta utilizando las tecnologías de la información y la comunicación. Una encuesta electrónica donde se ha recibido la información primaria. 
La encuesta se realizó a la comunidad Unadista del CEAD Pitalito. (Ver anexos 2, 3, 4). Allí se toma toda la información pertinente respecto a los tópicos en investigación y determinante para la formulación del PIGA.

- Toma de información en campo: en residuos sólidos, la caracterización de cada tipo de residuo, semanalmente dos veces, durante tres meses; en el recurso agua, análisis de la calidad de agua para consumo humano, estado actual del sistema, fugas, uso y manejo, número de llaves; en el recurso energía eléctrica, iluminación, número y tipo de bombillas, uso de equipos, uso de iluminación natural, estado actual del sistema, distribución; En el recurso insumos, tipo y número de insumos utilizados por la comunidad unadista, insumos de cafetería, de zonas verdes, reciclables, inservibles, orgánicos.

- Toma de información secundaria: las facturas de los servicios públicos de los recursos energía eléctrica, agua y residuos sólidos y la información suministrada por el almacén respecto a los insumos que la UNAD aporta al CEAD.

La información encontrada en los libros, revistas e internet, respecto a la normatividad ambiental.

\section{Análisis y evaluación de la situación ambiental}

De acuerdo con la información que suministran las técnicas de investigación, se concentra el esfuerzo en el análisis de la situación ambiental de la UNAD, CEAD Pitalito y su evaluación. Se realizó mediante el diagrama de redes de impactos, para cada una de los bloques designados, como el que se presenta en la figura 5.

Figura 5. Diagrama de identificación de impactos sobre el medio ambiente en la UNAD, CEAD Pitalito, 2009

ACCION EFECTO IMPACTO

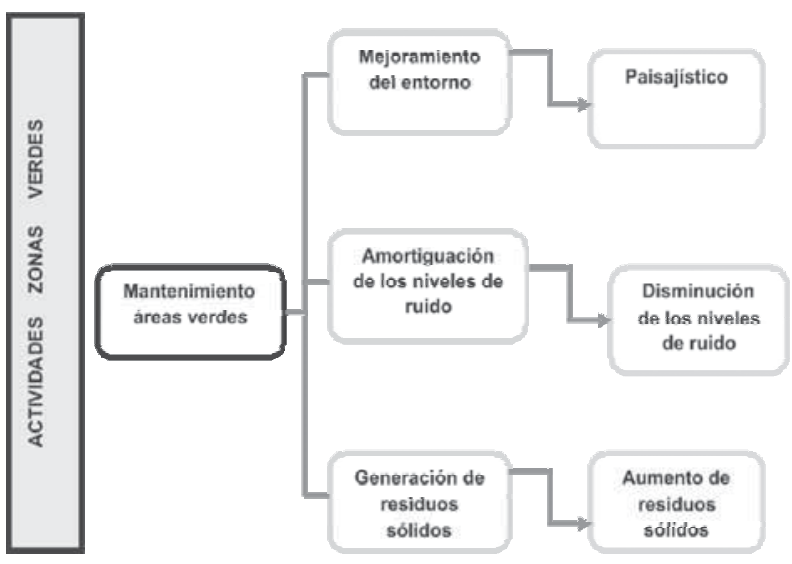

Fuente: Los autores 
Los diagramas de identificación permiten obtener una lista general de los impactos ambientales que pueden ser generados por una determinada actividad del proyecto, pero no indican nada sobre su significancia o jerarquía. Es por eso que en este paso se procede a valorarlos individualmente de tal forma que, con base en sus características más fácilmente identificables, se pueda determinar su trascendencia ambiental. Para ello se aplicará la expresión o índice denominado "Calificación ambiental" (Ca) de Arboleda, la cual dimensiona cinco criterios o factores característicos de cada impacto, según el Ministerio de Medio Ambiente: Clase (C), Duración (D), Evolución (E), Magnitud (M), Presencia (P).

De acuerdo con lo anterior, se utilizará el procedimiento propuesto por un grupo de empleados de las Empresas Públicas de Medellín, en la evaluación de impactos ambientales, por medio de un procedimiento completamente analítico, donde se desarrolló una ecuación de la calificación ambiental que permite obtener y explicar las relaciones de dependencia que existen entre los cinco criterios anteriores indicados, con el siguiente resultado:

Ca: C (P(ExM+D))

De acuerdo con la clasificación asignada individualmente a cada criterio, el valor absoluto de Ca será mayor que cero y menor o igual que 10. Este valor numérico se convierte luego en una expresión que indica la importancia del impacto (muy alta, alta, media, baja y muy baja) asignándole unos rangos con los resultados numéricos obtenidos. Con los resultados más altos, se priorizan los programas, los proyectos, las actividades, en la formulación del PIGA.

\section{RESULTADOS PARCIALES}

La tabla de respuestas, hasta ahora, permite apreciar el grado de desconocimiento de la legislación ambiental vigente, la necesidad de capacitación en este tema y un plan de gestión.

Estructura de la Gestión Ambiental externa. La construcción gradual y la institucionalización de mecanismos y roles de coordinación interinstitucional, a partir de necesidades y actividades concretas de gestión ambiental conjunta.

Mediante la participación en reuniones, foros, charlas, diálogo de saberes, comunicación escrita, internet, Se ha logrado la aceptación de la participación en la ejecución del PIGA, de los representantes de entidades oficiales y privadas de orden municipal, regional y nacional, de acuerdo con sus funciones y tareas específicas. Estos espacios, como la formulación del plan de ordenación y manejo de la cuenca hidrográfica del río Guarapas, la construcción colectiva del plan de educación ambiental y participación en el comité interinstitucional de educación ambiental CIDEA, Foro ambiental celebración de fechas ecológicas. Los compromisos y responsabilidades se presentan en la tabla 1. 
Tabla 1. Compromisos y responsabilidades para la gestión ambiental del PIGA UNAD CEAD Pitalito, 2010

\begin{tabular}{|c|c|c|c|}
\hline \multicolumn{4}{|c|}{ PIGA UNAD CEAD PITALITO. RESPONSABILIDADES GESTION AMBIENTAL } \\
\hline COMPONENTE & NECESIDAD & ACTIVIDAD & RESPONSABLE \\
\hline \multirow{2}{*}{$\begin{array}{l}\text { EDUCACION } \\
\text { AMBIENTAL }\end{array}$} & Socializar la normatividad & CIDEA & CAM - MUNICIPIO - UNAD \\
\hline & Promocionar los PIGA & Decreto municipal & Alcalde municipal \\
\hline \multirow{2}{*}{$\begin{array}{l}\text { FACTORES DE } \\
\text { RIESGO }\end{array}$} & \multirow{2}{*}{$\begin{array}{l}\text { Minimizar o eliminar } \\
\text { factores de riesgo }\end{array}$} & Contaminación visual & $\begin{array}{l}\text { Municipio de Pitalito, CAM, } \\
\text { UNAD }\end{array}$ \\
\hline & & Residuos sólidos & $\begin{array}{l}\text { EMPITALITO - Biorgánicos } \\
\text { del sur, CAM - UNAD }\end{array}$ \\
\hline \multirow{4}{*}{ RECURSOS } & \multirow{4}{*}{ Uso eficiente y ahorro } & Agua & $\begin{array}{l}\text { EMPITALITO - CAM - } \\
\text { UNAD }\end{array}$ \\
\hline & & Energía eléctrica & $\begin{array}{l}\text { Municipio de Pitalito, } \\
\text { Electrificadora del Huila, } \\
\text { UNAD }\end{array}$ \\
\hline & & Residuos sólidos & $\begin{array}{l}\text { Municipio de Pitalito, CAM, } \\
\text { UNAD }\end{array}$ \\
\hline & & Insumos & $\begin{array}{l}\text { CAM, Municipio de Pitalito, } \\
\text { UNAD }\end{array}$ \\
\hline
\end{tabular}

Fuente: Los autores

\section{Estructura de la Gestión Ambiental interna}

Tabla 2. Calificación ambiental de los impactos ambientales de la UNAD CEAD Pitalito, 2009

\begin{tabular}{|c|c|c|c|c|c|c|c|c|}
\hline RECURSOS & IIPACTOS & C.SE & PRESNCA: & Dtriaos & Evoltacias & MAGNIUD & Alificiad & MP ASBRIINTA \\
\hline \multirow{3}{*}{ AIRE } & Contaminación visual & $-0,2$ & 0,2 & 0.2 & 0,2 & 0.1 & 0,05 & MB \\
\hline & $\begin{array}{l}\text { Alteración y deterioro } \\
\text { de la calidad del aire }\end{array}$ & $-0,3$ & 0,3 & 0,5 & 0,3 & 0,3 & $-0,3$ & MB \\
\hline & Generación de Ruido & $-0,2$ & 0,3 & 0,5 & 0,3 & 0,3 & 0,22 & MB \\
\hline AGUA & \begin{tabular}{|l|} 
Aumento de \\
vertimientos liquidos al \\
alcantarillado
\end{tabular} & $\cdot 0,2$ & 0,3 & 0.3 & 0,3 & 0.3 & 0,14 & MB \\
\hline $\begin{array}{l}\text { RESIDUOS } \\
\text { SOLIDOS }\end{array}$ & $\begin{array}{l}\text { Incremento de residuos } \\
\text { solidos y mal manejo }\end{array}$ & -1 & 0,8 & 0,5 & 0,5 & 0,3 & 0,36 & MB \\
\hline ENERGIA & Perdida de energía & $-0,5$ & 0,5 & 0,4 & 0.8 & 0,8 & $-1,18$ & MB \\
\hline PAISAEE & $\begin{array}{l}\text { Calidad ambientaly } \\
\text { paisajistica }\end{array}$ & 0,5 & 0,5 & 0,4 & 0,4 & 0,3 & 0,8 & MB \\
\hline $\begin{array}{l}\text { EDUCACON } \\
\text { AMBIENTAL }\end{array}$ & $\begin{array}{l}\text { Qultura ciudadana e } \\
\text { institucional }\end{array}$ & 0,6 & 0,6 & 0,5 & 0,4 & 0,4 & 1,4 & MB \\
\hline
\end{tabular}

Fuente: Los autores 
La tabla permite visualizar que el impacto ambiental en la UNAD CEAD Pitalito es muy bajo, lo que corrobora los resultados anteriores.

Dado el desconocimiento de la legislación ambiental respecto a los recursos agua, energía eléctrica, residuos sólidos e insumos, a nivel interno - Comunidad universitaria - se formula el plan institucional de gestión ambiental - PIGA-, con programas para cada uno de los recursos, con un primer proyecto: Educación ambiental, apoyados por las entidades rectoras de cada uno de los recursos.

Con base en estas premisas, se ha firmado un pacto entre la empresa Aguas del Huila, S.A., E.S.P., y la UNAD CEAD Pitalito, dentro del programa departamental "Huila sin basuras" y se ha capacitado a la comunidad universitaria en este recurso y participación en la celebración del día del agua con la conferencia "Inventario sanitario rural", por parte de aguas del Huila y "Análisis de caudales en fuentes hídricas" por parte de la CAM.

Los resultados de las encuestas, se presentan en los anexos.

Se ha iniciado el proceso de consolidar el Comité paritario de salud ocupacional - COPASO- y el comité del PIGA.

\section{PERSPECTIVAS DEL TRABAJO}

Para el desarrollo del Plan de Gestión Ambiental PIGA, de la UNAD CEAD Pitalito, se implementará la "Estrategia de Educación Ambiental”. Se desarrollarán dos fases: una previa; que consiste en un programa de sensibilización y formación y una posterior de intervención.

- Programa de sensibilización y formación: consiste en el desarrollo de diferentes actividades educativas, lúdicas y pedagógicas que le permitan a cada individuo realizar un diagnóstico de su situación ambiental y de su responsabilidad social; no sólo en el campo laboral, sino desde todos los espacios donde se desempeñe al igual que los diferentes proyectos que genere. Sólo así, es posible generar individuos más críticos y reflexivos, que posicionen la dimensión ambiental en la escala de valores y lo asuman como una cuestión de bioética, que realmente genere cambios de hábitos, y les permita entender lo que implica una educación ambiental para el desarrollo sostenible.

- Diálogo de saberes y construcción de conocimiento: a partir de este proceso, se generarán espacios de diálogo entre la comunidad universitaria por escuelas y de escuelas diferentes, entre administrativos y estudiantes, para encontrar puntos de diferencia y puntos en común, permitiendo el diálogo de saberes y la construcción de conocimiento significativo colectivo (constructivismo social).

De la misma forma, estos espacios permiten desarrollar dinámicas de participación y concertación que pongan en contacto a los diferentes actores con una problemática ambiental común, la responsabilidad para tomar decisiones y que acompañados de bases conceptuales y normativas pertinentes, sean capaces de buscar soluciones, sin olvidar la 
gestión ambiental, que se convierte en la movilización de los diferentes actores capaces de hacer realidad sus planteamientos.

Finalmente, estos espacios facilitan a la comunidad educativa el conocimiento y apropiación de la entidad (clima organizacional), al igual, que fortalecer su proyección en espacios interinstitucionales, desde el reconocimiento de sus competencias y responsabilidades, de sus necesidades, aspiraciones e intereses tanto individuales como colectivos. Este proceso de formación en competencias ciudadanas nos permite la construcción de una cultura amigable con el ambiente, que a partir de la construcción de conocimiento significativo y la apropiación de realidades generen un manejo del ambiental consciente y responsable en el marco de la sostenibilidad.

- La replicabilidad de la metodología, tanto a nivel de CEAD, Ceres, CCAV de la UNAD, como a las entidades públicas y privadas desde lo local, a lo regional y nacional, como una apuesta de la UNAD, está dando resultados externos, como la asesoría a la Institución educativa Winnipeg, de Pitalito y a la Alcaldía del Municipio de San Agustín.

\section{DISCUSIÓN}

La UNAD, dado su cubrimiento a nivel nacional, puede y debe liderar el proceso de que una red social, mediante la capacitación y la acción, logre impactar en procesos de desarrollo todas las entidades oficiales formulen y ejecuten el Plan Institucional de Gestión Ambiental -PIGA-, y así contribuir de manera efectiva en el manejo adecuado de los recursos agua, energía eléctrica, residuos sólidos e insumos, de manera que permita una revolución social sostenible, que movilice a la comunidad en la protección, manejo, conservación y uso adecuado de los recursos naturales.

Si se logra, desde lo local e institucional, avanzar en esta propuesta, unida a la del sistema de Gestión Ambiental -SGA- de la UNAD, lograremos empoderar a esta Universidad como institución líder en procesos ambientales.

Es importante señalar que, aunque los Bloques de administración y aula múltiple tienen alrededor de casi 20 años de construidos, en general se encuentran en buen estado. Sin embargo, como recomendaciones a tener en cuenta en caso de remodelaciones, o adecuaciones a la infraestructura, el presente Plan identifica que se deben retomar en consideración los objetivos y criterios que se presentan aqui. 
ReVISTA DE INVESTIGACIONES UNAD

Tabla 3. Mejoramiento de las condiciones ambientales internas de la UNAD CEAD Pitalito, 2009

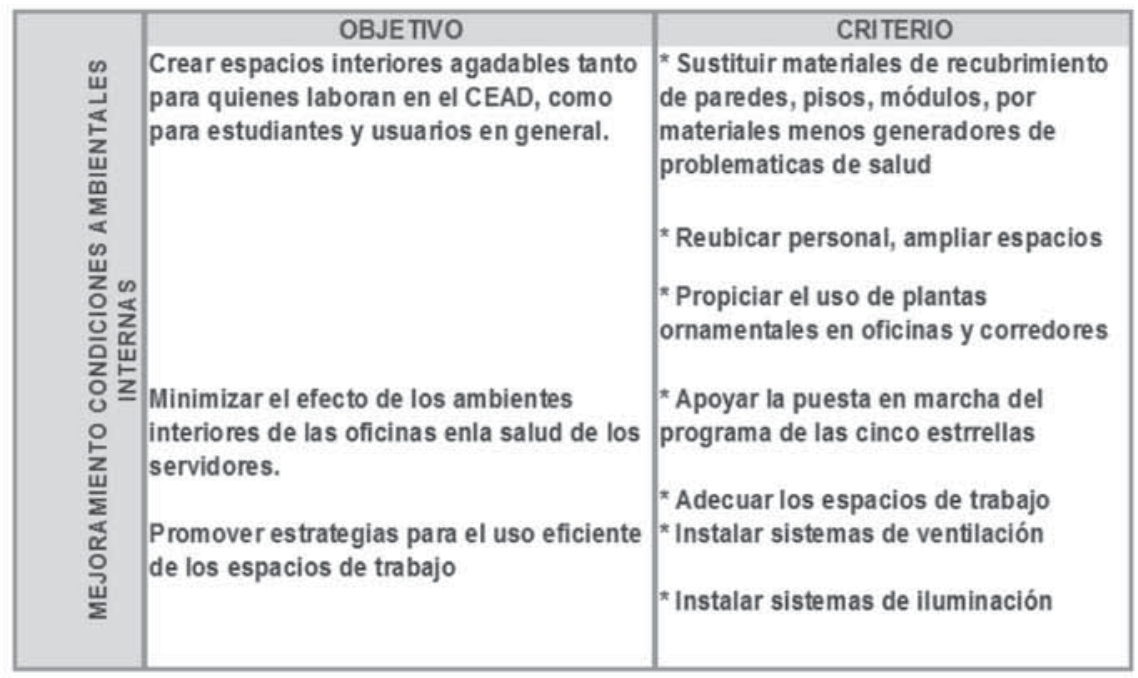

Fuente: los autores

\section{CONCLUSIONES}

La actual situación ambiental del planeta en general y de Colombia en particular, exige un compromiso de todas las instituciones y todas las personas para que aporten, desde su contexto, en aras de minimizar los impactos que sus acciones tienen sobre el desequilibrio de los ecosistemas mundiales.

La UNAD ha propuesto, dentro de su política ambiental, liderar estos procesos y ECAPMA CEAD Pitalito ha venido adelantando la formulación de una metodología que pueda ser replicable en los CEAD, CERES y CCAV de la UNAD. Lo hasta ahora encontrado, permite definir que los problemas ambientales se generan por el desconocimiento de la normatividad ambiental. La capacitación es la base del desarrollo armónico integral y del cambio cultural que se necesita.

Está abierto el debate para su continuo mejoramiento, para explorar procesos, proyectos exitosos, que generen una mayor participación tanto de la comunidad universitaria en su labor de liderazgo, como de toda la comunidad, como principal agente de todo proceso. 


\section{REFERENCIAS BIBLIOGRÁFICAS}

ALCALDÍA MAYOR DE BOGOTÁ. (2007), Departamento administrativo servicio civil Distrital, Plan Institucional de Gestión Ambiental, 35p.

ALCALDÍA MAYOR DE BOGOTÁ, D. C. (2005), Departamento técnico administrativo medio ambiente, oficina asesora de planeación, plan institucional de gestión ambiental programa de gestión ambiental interna-Dama, 33p.

ALCALDÍA MAYOR DE BOGOTÁ, D.C. (2006), Terminal de transporte, S.A., plan institucional de gestión ambiental (PIGA), 102 p.

ESCOBAR OCAMPO, DIEGO. (2006), Hospital Bosa II nivel. Empresa social del estado. Plan Institucional De Gestión Ambiental, Bogotá, 54p.

FUNDACIÓN UNIVERSAL. (2010), El edificio enfermo. En www.foment.com/ prevencion/.../prl/.../archivos\%5Cedificio.pdf consultado el el 28 marzo.

MÉNDEZ, ÁLVAREZ, CARLOS EDUARDO. Metodología, diseño y desarrollo del proceso de investigación. Editorial Mc Graw Hill. 2001. p.133.

MINISTERIO DEL AMBIENTE, vivienda y desarrollo territorial, Guía para selección de tecnologías de manejo integral de residuos sólidos, 2007.

MINISTERIO DEL AMBIENTE. MEIACOL. (1998), Manual de evaluación de impactos ambientales. $642 \mathrm{p}$.

MINISTERIO DE AMBIENTE. Vivienda y Desarrollo Territorial, Banco de documentos. En www.minambiente.gov.co, consulta realizada el 29 marzo de 2010.

MINISTERIO DE AMBIENTE. Vivienda y Desarrollo Territorial, Manual de evaluación de Impactos Ambientales en Colombia. En www.minambiente.gov.co, consulta realizada el 29 marzo de 2010.

MONROY, MANUEL M. Normas ISO 7730-1984. En http://editorial.cda.ulpgc.es/ ambiente/2_clima/7_comodo/index.htm, Consultado el 3 de Marzo de 2010.

MUNICIPIO DE PITALITO. Plan de desarrollo municipal, Periodo 2008-2011, Pitalito Huila.

MUNICIPIO DE PITALITO. Plan de ordenamiento territorial de Pitalito, Acuerdo 021 de 2000.

MONTEALEGRE T, WILLIAM I. (2009), Formulación del Plan Institucional de Gestión Ambiental para la UNAD CEAD Pitalito. Trabajo de grado. 


\section{ANEXOS}

\section{ANEXO 1.}

Normatividad ambiental vigente para la aplicabilidad de los PIGA, 2009

\begin{tabular}{|c|c|c|}
\hline VERTIMIENTOS & $\begin{array}{l}\text { Decreto } 1594 \text { de } \\
1984 \text { Minamblente }\end{array}$ & $\begin{array}{l}\text { Reglamantación sanitaria sobre usos del agua y } \\
\text { residuos líquidos. }\end{array}$ \\
\hline $\begin{array}{l}\text { GENERACION DE } \\
\text { RESIDUOS } \\
\text { PELIGROSOS }\end{array}$ & $\begin{array}{l}\text { Decreto } 2676 \text { de } \\
2000 \text { Minambiente }\end{array}$ & $\begin{array}{l}\text { Por el cual se reglamenta la Gestión Integral de } \\
\text { Residupos hospitalarios y similares }\end{array}$ \\
\hline \multirow{5}{*}{$\begin{array}{l}\text { EMISION FUENTES } \\
\text { MOVILES }\end{array}$} & Ley 769 de 2002 & $\begin{array}{l}\text { por el cuál se expide el nuevo código nacional del } \\
\text { transporte - certificado de gases }\end{array}$ \\
\hline & $\begin{array}{l}\text { Resolución } 005 \text { de } \\
1996 \text { y } 909 \text { de } 1996 \\
\text { de Min. Ambiente }\end{array}$ & $\begin{array}{l}\text { Por la cual se reglamentan los niveles de emisión } \\
\text { permisibles de contaminantes producidos por las } \\
\text { fuentes móviles. }\end{array}$ \\
\hline & $\begin{array}{l}\begin{array}{l}\text { Decreto } 948 \mathrm{de} \\
1995\end{array} \\
\end{array}$ & $\begin{array}{l}\text { Contiene el Reglamento de Protección y Control de la } \\
\text { Calidad del Aire. }\end{array}$ \\
\hline & $\begin{array}{l}\text { Decreto } 2107 \text { de } \\
1995\end{array}$ & $\begin{array}{l}\text { Por me dio del cual se modifica parcialmente el } \\
\text { Decreto } 948 / 95 \text { Reglamento de Protección y Control } \\
\text { de la Calidad del Aire. }\end{array}$ \\
\hline & $\begin{array}{l}\text { Resolución } 556 \text { de } \\
2006\end{array}$ & $\begin{array}{l}\text { Por el cual se expiden las normas para el control de } \\
\text { emisiones de fuenets móviles }\end{array}$ \\
\hline \multirow[b]{2}{*}{ RUIDO } & $\begin{array}{l}\text { Res } 1792 \text { de } 1990 \\
\text { Min trabajo y } \\
\text { Seguridad social }\end{array}$ & $\begin{array}{l}\text { Por el cual se adoptan valores límites permisibles } \\
\text { para la exposición ocupacional al ruido }\end{array}$ \\
\hline & $\begin{array}{l}\text { Resolución } 8321 \text { de } \\
1983 \text { Min. Salud }\end{array}$ & $\begin{array}{l}\text { Por la cual se dictan nomas sobre Protección y } \\
\text { Conservación de la Audición de la Salud y el } \\
\text { bienestar de las personas, por causa de la } \\
\text { producción yemisión de nuidos. }\end{array}$ \\
\hline $\begin{array}{l}\text { TRANSPORTE DE } \\
\text { RESIDUOS } \\
\text { PELIOROSOS }\end{array}$ & $\begin{array}{l}\text { Decreto } 1609 \text { de } \\
2002\end{array}$ & $\begin{array}{l}\text { Por el cual se reglamenta el manejo y transporte } \\
\text { terrestre automotor de mercancias peligrosas por } \\
\text { carretera }\end{array}$ \\
\hline \multirow{3}{*}{$\begin{array}{l}\text { PRESTACION DEL } \\
\text { SERVICIO PUBLICO } \\
\text { DE ASEO }\end{array}$} & $\begin{array}{l}\text { Decreto } 1713 \text { de } \\
2002\end{array}$ & $\begin{array}{l}\text { Por el cual se reglamenta la ley } 142 \text { de } 1994 \\
\text { prestación del servico público de aseo - presentación } \\
\text { de los R.S. }\end{array}$ \\
\hline & Códiao de policia & RE-RE-Separación en la fuente \\
\hline & Ley 9 de 1979 & $\begin{array}{l}\text { Código sanitario nacional - Instalaciones adecuadas } \\
\text { para almacenamiento de R.S. }\end{array}$ \\
\hline \multirow{4}{*}{$\begin{array}{l}\text { INSTALACIONES } \\
\text { ELECTRICAS }\end{array}$} & Ley 143 de 1994 & $\begin{array}{l}\text { Adecuado mantenimiento de instalaciones eléctricas } \\
\text { riesgos, a horro y uso eficiente de energia. }\end{array}$ \\
\hline & $\begin{array}{l}\text { Decreto } 2331 \text { de } \\
2007\end{array}$ & Sistema de ahorro de energía - RETIE - NTC 2050 \\
\hline & $\begin{array}{l}\text { Ley } 697 \\
\text { de } 2001\end{array}$ & $\begin{array}{l}\text { Mediante la cual se fomenta el uso racional y } \\
\text { eficiente de la energía, se promueve la utilización de } \\
\text { energias altemativas y se dictan otras disposiciones }\end{array}$ \\
\hline & $\begin{array}{l}\text { Decreto } 3683 \text { de } \\
2003\end{array}$ & $\begin{array}{l}\text { Por el cual se reglamenta la Ley } 697 \text { de } 2001 \text {, sobre } \\
\text { el uso racional y eficiente de la energía. }\end{array}$ \\
\hline \multirow{4}{*}{ AGUA } & Ley 373 de 1997 & $\begin{array}{l}\text { Uso eficiente y ahorro del agua - Instalación de } \\
\text { equipos. }\end{array}$ \\
\hline & $\begin{array}{l}\text { Resolución } 1433 \text { de } \\
\text { 2004, MAVDT }\end{array}$ & $\begin{array}{l}\text { Reglamenta el artículo } 12 \text { del Decreto } 3100 \text { de } 2003, \\
\text { sobre Planes de Saneamiento y Manejo de } \\
\text { vertimientos. PSIMV }\end{array}$ \\
\hline & $\begin{array}{l}\text { Decreto } 475 \text { de } \\
1998\end{array}$ & Normas técnicas de calidad del agua potable. \\
\hline & $\begin{array}{l}\text { Decreto } 3102 \text { de } \\
1997\end{array}$ & $\begin{array}{l}\text { Obligación de reparar y reemplazar instalaciones que } \\
\text { presenten fugas }\end{array}$ \\
\hline
\end{tabular}

Fuente: www.minambiente.gov.co/normatividad 


\section{ANEXO 2.}

Organización bloques en la UNAD, CEAD Pitalito, 2010

\begin{tabular}{|c|l|c|l|c|l|}
\hline Bloque & \multicolumn{1}{|c|}{ Área } & Área $\mathbf{M}^{2}$ & \multicolumn{1}{|c|}{$\begin{array}{c}\text { Dependencias que } \\
\text { funcionan }\end{array}$} & Personal & \multicolumn{1}{|c|}{ Servicios } \\
\hline 1 & Administración & 328 & $\begin{array}{l}\text { Dirección, secretaria, conseje- } \\
\text { ría, sistemas, sala de tutores. }\end{array}$ & 33 & $\begin{array}{l}\text { Atención al públicoy estudian- } \\
\text { tes, baños administrativos }\end{array}$ \\
\hline 2 & Aula máxima & 1096 & Cafetería, aula máxima, & 2 & $\begin{array}{l}\text { Sala de reuniones, cafete- } \\
\text { ría, baños. }\end{array}$ \\
\hline 3 & Aulas estudio & 210 & Aulas de estudio & 833 & Estudio \\
\hline 4 & Zonas públicas & 5481.62 & Parqueadero, zona verde & 120 & Parqueadero, seguridad \\
\hline
\end{tabular}

Fuente: los autores

ANEXO 3.

Rango de valoración de los criterios de evaluación usado por el método de EEPPM para la evaluación de impactos ambientales

\begin{tabular}{|c|c|c|}
\hline CRITERIO & RANGO & VALOR (1) \\
\hline CLASE & $\begin{array}{l}\text { Positivo (t) } \\
\text { Negativo (c) }\end{array}$ & \\
\hline PRESENCLR & $\begin{array}{l}\text { Cierta } \\
\text { Muy probable } \\
\text { Probable } \\
\text { Poco probable } \\
\text { No probable }\end{array}$ & $\begin{array}{l}1,0 \\
0.7 \bullet 0.99 \\
0,3<0,69 \\
0,1 \cup 0,29 \\
0,0 \cup 0,09\end{array}$ \\
\hline DURACION & $\begin{array}{l}\text { Muy larga o permanente: Si es > de } 10 \text { años } \\
\text { Langa: Si es > de } 7 \text { años } \\
\text { Media: Si es > de } 4 \text { años } \\
\text { Corta: Si es > de } 1 \text { año } \\
\text { Muy corta: Si es < de } 1 \text { año }\end{array}$ & $\begin{array}{l}1,0 \\
0,7<0,99 \\
0,4<0,89 \\
0,1<0,39 \\
0,0<0,09\end{array}$ \\
\hline EVOLUCION & $\begin{array}{l}\text { Muy rapida: Si es < de } 1 \text { mes } \\
\text { Rápida: Si es < de } 12 \text { meses } \\
\text { Madia: Si cs < de } 18 \text { mocos } \\
\text { Lenta: Si es < de } 24 \text { meses } \\
\text { Muy lenta: Si es > de } 24 \text { meses }\end{array}$ & $\begin{array}{l}0,8 \leq 1,0 \\
0,6<0,79 \\
0,1<0,60 \\
0,2<0,39 \\
0,0<0,19\end{array}$ \\
\hline MRGNITUD & $\begin{array}{l}\text { Muy alta: Si M }(2)>\text { de } 80 \% \\
\text { Ata: Si M vańa entre } 60 \text { y } 80 \text { \% } \\
\text { Media: Si Mr varia entre } 40 \text { y } 60 \% \\
\text { Baja: Si Mr varía entre } 20 \text { y } 40 \% \\
\text { Muy baja: Si Mr < del } 20 \%\end{array}$ & $\begin{array}{l}0,8 \leq 1,0 \\
0,6<0,79 \\
0,4<0,59 \\
0,2<0,39 \\
0,0<0,19\end{array}$ \\
\hline $\begin{array}{l}\text { IMPORTANCIR } \\
\text { AMBIENTAL }\end{array}$ & $\begin{array}{l}\text { Muy alta: Si Ca varia entre } \\
\text { Ata: Si Ca varia entre } \\
\text { Media: Si Ca varia entre } \\
\text { 日aja: Si Ca varía entre } \\
\text { Muy baja: Si Ca varía entre }\end{array}$ & $\begin{array}{l}8,0 \leq 10,0 \\
6,0<7,9 \\
4,0<5,9 \\
2,0<3,8 \\
0,0<1,9\end{array}$ \\
\hline $\begin{array}{l}\text { CONSTANTES } \\
\text { DE } \\
\text { PONDERACION }\end{array}$ & $\mathrm{a}$ & $\begin{array}{l}a=7,0 \\
b=3,0\end{array}$ \\
\hline
\end{tabular}

Fuente: EEPPM, 2007 
ANEXO 4.

Resultados de la encuesta a cuerpo académico y administrativo CEAD Pitalito, componentes atmosférico, 2010

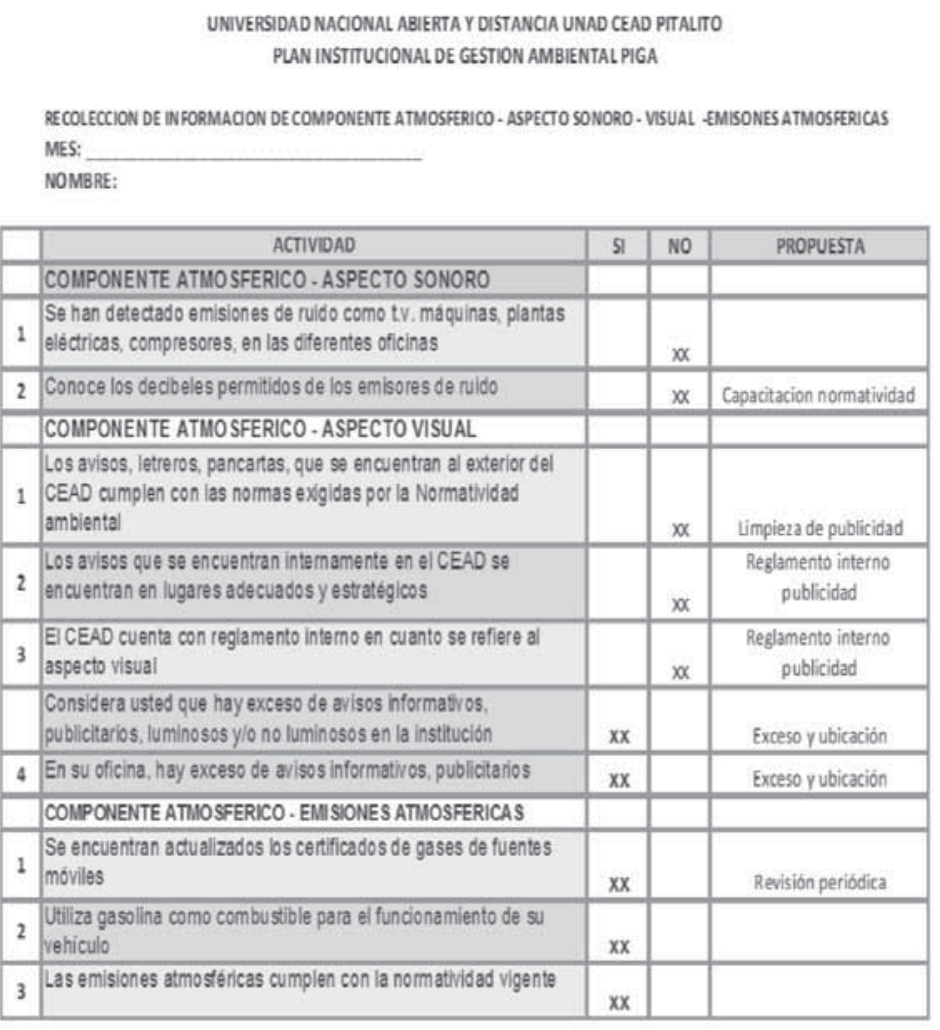

Fuente: Los autores 


\section{ANEXO 5. \\ Resultado de la encuesta a UNAD CEAD Pitalito, respecto al uso eficiente y ahorro del agua, 2010}

\begin{tabular}{|c|c|c|c|}
\hline \multicolumn{4}{|c|}{$\begin{array}{l}\text { UNIVERSIDAD NACIONAL ABIERTA Y DISTANCIA UNAD CEAD PITALITO } \\
\text { PLAN INSTITUCIONAL DE GESTION AMBIENTAL PIGA }\end{array}$} \\
\hline \multicolumn{4}{|l|}{$\begin{array}{l}\text { RECOLECCION INFORMACION DE CONSUMO DE AGUA } \\
\text { FECHA } \\
\text { NOMBRE: }\end{array}$} \\
\hline ACTIVIDAD & SI & NO & PROPUESTA \\
\hline SE CONOCE EL CONSUMO DE AGUA MENSUAL & & $\mathrm{xx}$ & CAPACITACION \\
\hline SE CHEQUEA LA CALIDAD DEL AGUA DE SUMINISTRO & & $\mathrm{xX}$ & CAPACITACION \\
\hline EXISTE UN PROGRAMA DE AHORRO DEL AGUA & & $x x$ & PIGA \\
\hline $\begin{array}{l}\text { SE TIENEN CARTELES CERCA DE LAS LLAVES RECORDANDO } \\
\text { EL AHORRO DEL AGUA }\end{array}$ & & $x x$ & CARTELES \\
\hline $\begin{array}{l}\text { SE HAN TOMADO ACCONES ESPECIFICAS PARA AHORRAR } \\
\text { AGUA EN LOS ULTIMOS DOCE MESES }\end{array}$ & & $x x$ & PIGA \\
\hline $\begin{array}{l}\text { SE REPORTAN INMEDIATAMENTE LAS FUGAS DE AGUA DE } \\
\text { SANITARIOS, LAVAMANOS, LAVES. }\end{array}$ & $x x$ & & $\begin{array}{l}\text { MANUAL PROCESOS Y } \\
\text { PROCEDIMIENTOS }\end{array}$ \\
\hline $\begin{array}{l}\text { SE REPARAN OPORTUNAMENTE LAS FU GAS UNA VEZ SE } \\
\text { INFORMA EL DAÑO }\end{array}$ & & $x x$ & CAJA MENOR \\
\hline SE MANTIENEN CERRADOS LOS GRIFOS CUANDO NO SE & $x x$ & & INSPECCION PERIODICA \\
\hline $\begin{array}{l}\text { SE TIENEN INSTALADOS LAVAMANOS Y SANITARIOS QUE } \\
\text { SE ACTIVEN SEGÚN SU UTILIZACION Y/O SEAN }\end{array}$ & $x x$ & & \\
\hline $\begin{array}{l}\text { SE HA INTENTADO REDUAR EL VOLUMEN DE LA } \\
\text { DESCARGA DE LOS SANITARIOS CON OBJETOS COMO }\end{array}$ & & $x x$ & REDUCCION \\
\hline $\begin{array}{l}\text { SE HA ESTUDIADO EN QUE LUGARES SE PUEDE REUTILIZAR } \\
\text { EL AGUA Y EN QUE CANTIDAD }\end{array}$ & & $x x$ & PLAN DE AGUAS LUUVIAS \\
\hline $\begin{array}{l}\text { SE RECOLECTA EL AGUA LLUVIA EN TANQUES PARA SER } \\
\text { UTILIZADA EN ALGUNA ACTIVIDAD OSE HA CONSIDERADO }\end{array}$ & & $x x$ & PLAN DE AGUAS LLUVIAS \\
\hline EXISTEN FUGAS DE AGUA & SI & & INSPECCION PERIODICA \\
\hline DONDE & & & LAVAMANOS \\
\hline
\end{tabular}


ANEXO 6.

Resultados encuesta a cuerpo académico y administrativo sobre uso y manejo recurso energía, UNAD, CEAD Pitalito, 2010

\begin{tabular}{|c|c|c|c|}
\hline ACTIVIDAD & SI & NO & PROPUESTA \\
\hline $\begin{array}{l}\text { Se sabe cuanta energia se consume en total, cuanta en las } \\
\text { diferentes áreas, cuanta en su oficina. }\end{array}$ & & $x x$ & CAPACITACION \\
\hline Se han fijado objetivos para reducir el consumo de energia & & $x x$ & $\begin{array}{l}\text { PROGRAMARREDUCCION } \\
\text { CONSUMO }\end{array}$ \\
\hline Existe un programa de ahorro de energía & & $x x$ & $\begin{array}{l}\text { PROGRAMA REDUCCION } \\
\text { CONSUMO }\end{array}$ \\
\hline Se usan fuentes de energia mas económicas & & $\mathrm{xx}$ & CAIA MENOR \\
\hline $\begin{array}{l}\text { Se verifica el consumo de energia de los aparatos eléctricos antes } \\
\text { de comprarios }\end{array}$ & & $\mathrm{xx}$ & $\begin{array}{l}\text { MANUAL PROCESOSY } \\
\text { PROCEDIMIENTOS }\end{array}$ \\
\hline $\begin{array}{l}\text { Se realiza un mantenimiento perióoico programado para los } \\
\text { equipos }\end{array}$ & & $x x$ & $\begin{array}{l}\text { MANUAL PROCESOSY } \\
\text { PROCEDIMIENTOS }\end{array}$ \\
\hline $\begin{array}{l}\text { Se tienen temporizadores para los equipos de modo que se } \\
\text { apaguen cuando no son utilizados por un periodo determinado }\end{array}$ & & $\mathrm{xx}$ & $\begin{array}{l}\text { RECONSTRUCCION LINEASY } \\
\text { EQUIPOS }\end{array}$ \\
\hline $\begin{array}{l}\text { Se han utilizado equipos de control: Sistemas de manejo de energia } \\
\text { en tiempo real, temporizadores, celdas fotoelectricas, otros, } \\
\text { ¿Cuales? }\end{array}$ & & $\mathbf{x x}$ & $\begin{array}{l}\text { RECONSTRUCCION UINEASY } \\
\text { EQUIPOS }\end{array}$ \\
\hline Se ha instalado luminación de bajo consumo de energia & & $\mathrm{xx}$ & CAIA MENOR \\
\hline Se encienden las luces sólo cuando la luz natural es deficiente & $x x$ & & \\
\hline $\begin{array}{l}\text { Se limpian con frecuencia las lámparas y el sistema de liuminación } \\
\text { para mejorar la radiación y la capacidad de lluminación }\end{array}$ & & $\mathrm{xx}$ & CAIA MENOR \\
\hline $\begin{array}{l}\text { Se tiene instaladas láminas traslúcidas para aprovechar la } \\
\text { iluminación natural }\end{array}$ & & $\mathrm{xx}$ & MANTENIMIENTOSEDE \\
\hline $\begin{array}{l}\text { Se apagan las luces y los equipos (computadores) en las oficinas } \\
\text { desocupadas }\end{array}$ & $x x$ & & $\begin{array}{l}\text { MANUAL PROCESOSY } \\
\text { PROCEDIMIENTOS }\end{array}$ \\
\hline $\begin{array}{l}\text { Se apagan las luces de corredores y áreas comunes temprano en la } \\
\text { noche }\end{array}$ & $x x$ & & MONTOREO \\
\hline
\end{tabular}




\section{ANEXO 7.}

Facturación servicios de energía eléctrica UNAD, CEAD Pitalito, 2009

\begin{tabular}{|l|l|c|c|c|c|}
\hline \multirow{2}{*}{ AÑO } & \multirow{2}{*}{ MES } & \multicolumn{2}{c|}{ CONSUMO } & \multirow{2}{*}{$\begin{array}{c}\text { SUBTOTAL } \\
\text { CONSUMO }\end{array}$} & \multirow{2}{*}{ VALOR } \\
\cline { 3 - 4 } & & CONTADOR 1 & CONTADOR 2 & \\
\hline 2009 & ABRIL & 786 & 103 & 889 & 333721,71 \\
\hline 2009 & MAYO & 820 & 116 & 936 & 351365,04 \\
\hline 2009 & JUNIO & 1067 & 160 & 1227 & 460603,53 \\
\hline 2009 & JULIO & 1122 & 158 & 1280 & 480499,2 \\
\hline 2009 & AGOSTO & 1021 & 155 & 1176 & 441458,64 \\
\hline 2009 & SEPTIEMBRE & 1182 & 188 & 1370 & 514284,3 \\
\hline 2009 & OCTUBRE & 550 & 102 & 652 & 244754,28 \\
\hline 2009 & NOVIEMBRE & 928 & 160 & 1088 & 408424,32 \\
\hline SUBTOTAL & & 7476,00 & 1142,00 & 8618 & 3235111,02 \\
\hline PROMEDIO & & 934,50 & 142,75 & 1077,25 & 404388,88 \\
\hline
\end{tabular}

Fuente: los autores - Electrificadora del Huila

ANEXO 8.

Resultado encuesta a UNAD CEAD Pitalito sobre residuos sólidos, 2010

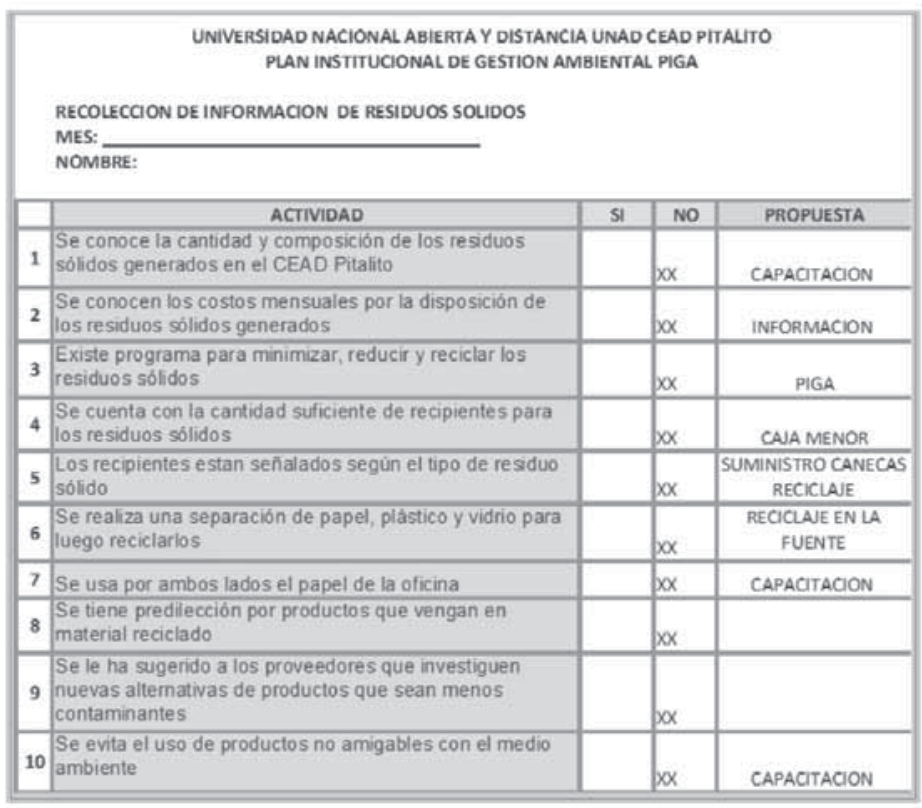

Fuente: los autores 


\section{ANEXO 9.}

Recipientes para residuos sólidos UNAD, CEAD Pitalito, 2009

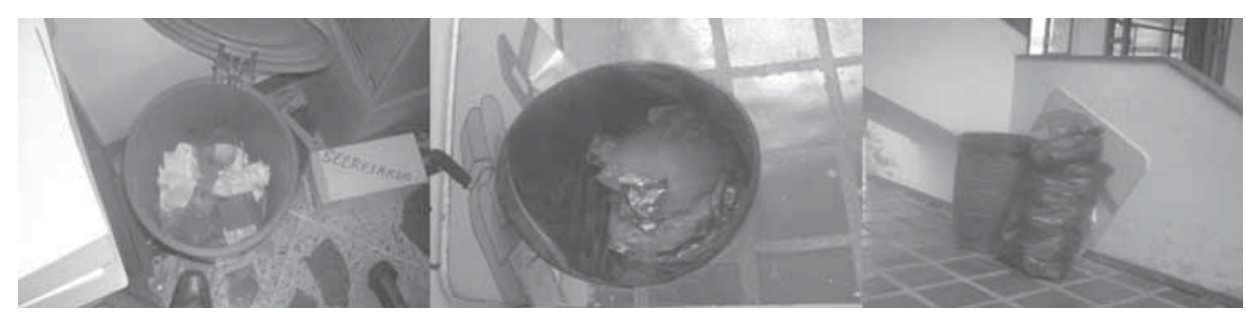

ANEXO 10.

Caracterización (producción y composición) de los residuos sólidos UNAD, CEAD Pitalito, 2009

PLAN INSTITUCIONAL DE GESTIÓN AMBIENTAL PIGA

RECOLECCIÓN DE INFORMACIÓN DE ENTREGA DE RESIDUOS

MES

NOMBRE:

\begin{tabular}{|c|c|c|c|c|c|c|r|}
\hline FECHA & PAPEL $(\mathbf{K g})$ & CARTÓN $(\mathbf{K g})$ & VIDRIO $(\mathbf{K g})$ & PLÁSTICO $(\mathbf{K g})$ & OTROS $(\mathbf{K g})$ & TOTAL $\mathbf{( K g})$ & ENTREGADO A \\
\hline $05 / 09 / 2009$ & 23 & 4 & 8 & 12 & 6 & 53 & EMPITALITO \\
\hline $12 / 09 / 2009$ & 18 & 6 & 6 & 14 & 4 & 48 & EMPITALITO \\
\hline $19 / 09 / 2009$ & 12 & 8 & 12 & 8 & 3 & 43 & EMPITALITO \\
\hline $26 / 09 / 2009$ & 12 & 4 & 8 & 8 & 6 & 38 & EMPITALITO \\
\hline $03 / 10 / 2009$ & 14 & 6 & 12 & 6 & 8 & 46 & EMPITALITO \\
\hline $10 / 10 / 2009$ & 3 & 3 & 4 & 3 & 12 & 25 & EMPITALITO \\
\hline $17 / 10 / 2009$ & 4 & 5 & 4 & 3 & 5 & 21 & EMPITALITO \\
\hline $24 / 10 / 2009$ & 6 & 5 & 7 & 6 & 8 & 32 & EMPITALITO \\
\hline $31 / 10 / 2009$ & 5 & 6 & 3 & 3 & 5 & 22 & EMPITALITO \\
\hline $07 / 11 / 2009$ & 12 & 6 & 6 & 6 & 8 & 38 & EMPITALITO \\
\hline $14 / 11 / 2009$ & 14 & 8 & 10 & 6 & 4 & 42 & EMPITALITO \\
\hline SUBTOTAL & 123 & 61 & 80 & 75 & 69 & 408 & \\
\hline PROMEDIO & 11.18 & 5.55 & 7.27 & 6.82 & 6.27 & 37.0909 & \\
\hline Fu & & & & &
\end{tabular}

Fuente: Los autores 


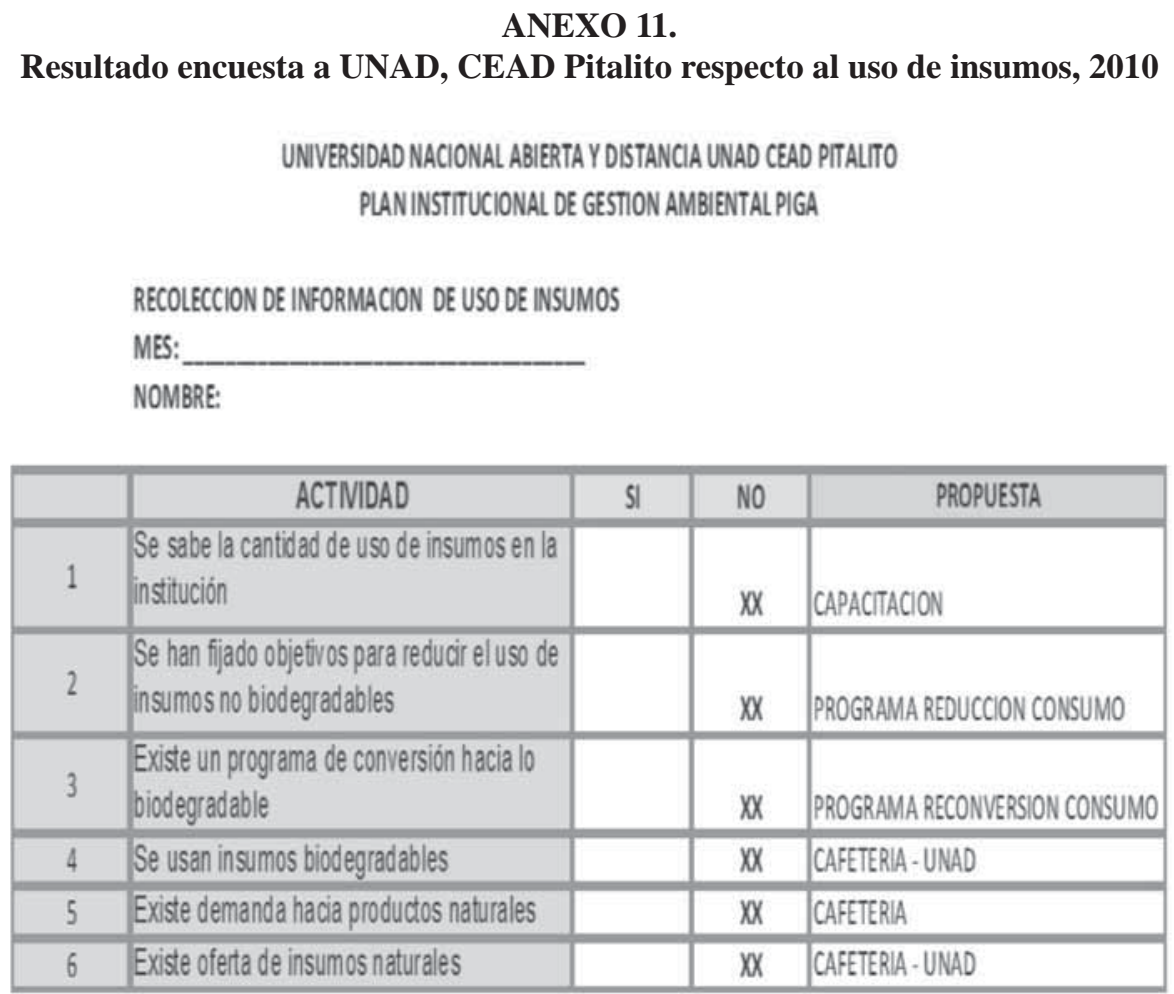

Fuente: Los autores 